\title{
Caracterização genética de raças caprinas nativas brasileiras utilizando-se 27 marcadores microssatélites ${ }^{1}$
}

\author{
Marcos Paulo Carrera Menezes ${ }^{2}$, Amparo Martinez Martinez ${ }^{3}$, Maria Norma Ribeiro ${ }^{4}$, Edgard \\ Cavalcanti Pimenta Filho5, Juan Vicente Delgado Bermejo ${ }^{3}$
}

\footnotetext{
${ }^{1}$ Parte da tese de Doutorado do primeiro autor do Programa de Doutorado Integrado em Zootecnia - PDIZ/UFPB.

2 Doutorando da Universidade Federal da Paraíba - PDIZ - Areia - PB.

${ }^{3}$ Departamento de Genética - Universidade de Córdoba - Córdoba - Espanha.

${ }^{4}$ Universidade Federal Rural de Pernambuco - PDIZ - Recife - PE.

${ }^{5}$ Universidade Federal da Paraíba - PDIZ - Areia - PB.
}

RESUMO - Objetivou-se com este trabalho verificar a utilização de 27 microssatélites para caracterização genética das raças caprinas nativas do Brasil. Foram coletadas amostras de pêlos de 332 animais das raças Moxotó (60), Canindé (50), Serrana Azul (55), Marota (68), Repartida (52) e Graúna (47). Selecionaram-se 27 microssatélites, que foram amplificados mediante a técnica de reação em cadeia de polimerase (PCR). Os produtos amplificados foram submetidos à eletroforese em gel de poliacrilamida, em um seqüenciador automático ABI 377XL. Todos os microssatélites analisados mostraram-se polimórficos para o estudo de caracterização genética das raças nativas brasileiras. O número de alelos variou de 3 (MAF209) a 23 (CSSM66). A heterozigosidade média esperada foi de 0,672 e a observada, de 0,582. Todos os microssatélites analisados foram informativos, à exceção do marcador MAF209 (PIC: 0,042). Os microssatélites analisados mostraram-se polimórficos e os resultados obtidos indicaram a capacidade desses marcadores em identificar a variabilidade genética em raças caprinas nativas brasileiras.

Palavras-chave: caprinos, conservação genética, marcadores moleculares, variabilidade genética

\section{Genetic characterization of Brazilian native breeds of goats using 27 markers microsatellites}

\begin{abstract}
This study aimed to evaluate 27 microsatellites for genetic characterization of Brazilian native goats using samples of hair collected from 332 animals of the Moxotó, Canindé, Serrana Azul, Marota, Repartida and Graúna breeds. Twenty-seven microsatellites were selected, amplified by polymerase chain reaction (PCR). The amplified products were submitted to electrophoreses in poliacrylamide gel using an ABI 377XL automatic sequencer. The number of alleles ranged between three (MAF209) and twenty-three (CSSM66). Averages of expected and observed heterozigosity were respectively 0,672 and 0,582. Except for the marker MAF209 (PIC: 0,042), all the analyzed microsatellites were informative. The polymorphism of all microsatellites indicate their hability in identifying genetic variability of Brazilian native breeds of goats.
\end{abstract}

Key Words: goats, genetic conservation, molecular markers, genetic variability

\section{Introdução}

As raças caprinas nativas do Brasil estão localizadas na Região Nordeste, onde os animais são criados em sistemas extensivo e semi-extensivo para produção de carne, pele e leite. São animais bem adaptados à região semi-árida e resistentes a doenças e parasitas. Porém, são poucos os trabalhos realizados com esses animais, ameaçados de extinção. Para minimizar esse problema, um programa de conservação foi estruturado e uma de suas prioridades é o estudo da caracterização genética desses patrimônios genéticos.
Segundo Gama (2004), a variabilidade genética total das espécies é representada pela contribuição das variabilidades inter e intra-raciais. Verifica-se, portanto, a importância de se medir a variabilidade genética dos animais, visto que a conservação dos recursos genéticos está efetivamente relacionada à manutenção das variabilidades inter-racial (evita a extinção das raças) e intra-racial (evita a erosão genética).

A caracterização genética com o uso de marcadores moleculares tem demonstrado ser uma ferramenta eficaz para quantificação da diversidade genética de animais domésticos. Os marcadores genéticos são loci que apre- 
sentam características detectáveis que diferenciam os indivíduos de determinada população, demonstrando variações entre indivíduos e grupos de animais.

Os microssatélites são pequenas sequências repetidas (1 a 6 nucleótideos), abundantes, distribuídas ao acaso por todo o genoma. Apresentam grande polimorfismo, são de fácil identificação e têm sido utilizados em mapas de ligação em mamíferos, identificação individual em controle de paternidade e caracterização genética de populações.

Segundo a FAO, os microssatélites que possuem homologia em várias espécies relacionadas são preferíveis, pois facilitam o trabalho em laboratório e possibilitam economia de tempo e redução dos custos.

Yang et al. (1999) analisaram seis microssatélites em quatro raças de caprinos chineses e demonstraram que os microssatélites de ovinos (OarFCB11, OarFCB20, OarFCB304, OarFCB48 e Oar FCB34) e bovinos (BM4621) são eficientes para detectar polimorfismo em raças caprinas. Ainda com caprinos nativos chineses, Li et al. (2002) tipificaram 16 microssatélites recomendados pela $E U$ Sheep and Goat Biodiversity Project. A variabilidade genética intra e inter-racial em caprinos asiáticos nativos foi verificada por Barker et al. (2001), com 25 microssatélites. Saitbekova et al. (1999) realizaram um estudo sobre a diversidade genética entre raças caprinas suíças, no qual analisaram 20 microssatélites de bovinos. O objetivo neste trabalho foi verificar a possibilidade de utilização de 27 microssatélites para caracterização genética das raças caprinas brasileiras.

\section{Material e Métodos}

Foram coletadas 332 amostras casualizadas de pêlos de caprinos das raças Moxotó (60), Canindé (50), Serrana Azul (55), Marota (68), Repartida (52) e Graúna (47) de vários rebanhos e estados distintos da Região Nordeste do Brasil (Paraíba, Pernambuco, Rio Grande do Norte, Bahia e Piauí). O DNA das amostras foi extraído de dez pêlos selecionados por animal, segundo metodologia estabelecida por Walsh etal. (1991).

Na Tabela 1 são apresentados os 27 microssatélites analisados com as sequências direta e reversa dos iniciadores (Primers). Os multiplex, os tamanhos dos fragmentos, os fluorocromos e a temperatura de anelamento dos loci encontram-se na Tabela 2.

Os microssatélites foram amplificados mediante a técnica de reação em cadeia de polimerase (PCR), com $25 \mu \mathrm{L}$ de volume final de uma reação: $5 \mu \mathrm{L}$ de DNA, $2,5 \mathrm{mM}$ de cloreto de magnésio, 1 unidade de Taq Polimerase, $200 \mathrm{mM}$ de

Tabela 1 - Microssatélites analisados com as respectivas seqüências (direta e reversa) dos iniciadores

Table 1 - Analyzed microsatellites and respective sequences (direct and reverse) of primers

\begin{tabular}{|c|c|c|}
\hline locus & $\begin{array}{c}\text { Direta } \\
\text { Direct }\end{array}$ & $\begin{array}{c}\text { Reversa } \\
\text { Reverse }\end{array}$ \\
\hline BM6506 & GCACGTGGTAAAGAGATGGC & AGCAACTTGAGCATGGCAC \\
\hline BM8125 & СТСТАTСТGTGGAAAAGGTGGG & GGGGGTTAGACTTCAACATACG \\
\hline BM1818 & AGCTGGGAATATAACCAAAGG & AGTGCTTTCAAGGTCCATGC \\
\hline CSRD247 & GGACTTGCCAGAACTCTGCAAT & CACTGTGGTTTGTATTAGTCAGG \\
\hline ЕTH225 & GATCACCTTGCCACTATTTCCT & ACATGACAGCCAGCTGCTACT \\
\hline HAUT 27 & TTTTATGTTCATTTTTTGACTGG & AACTGCTGAAATCTCCATCTTA \\
\hline ILSTS011 & GCTTGCTACATGGAAAGTGC & CTAAAATGCAGAGCCCTACC \\
\hline INRA63 & ATTTGCACAAGCTAAATCTAACC & AAACCACAGAAATGCTTGGAAG \\
\hline INRA5 & TTCAGGCATACCCTACACCACATG & AAATATTAGCCAACTGAAAACTGGG \\
\hline SPS 115 & AAAGTGACACAACAGCTTCTCCAG & AACGAGTGTCCTAGTTTGGCTGTG \\
\hline TGLA 122 & СССТССТССAGGTAAATCAGC & AATCACATGGCAAATAAGTACATAC \\
\hline BM6526 & CATGCCAAACAATATCCAGC & TGAAGGTAGAGAGCAAGCAGC \\
\hline CSRM60 & AAGATGTGATCCAAGAGAGAGGCA & AGGACCAGATCGTGAAAGGCATAG \\
\hline CSSM66 & ACACAAATCCTTTCTGCCAGCTGA & AATTTAATGCACTGAGGAGCTTGG \\
\hline ETH 10 & GTTCAGGACTGGCCCTGCTAACA & СCTCCAGCCCACTTTCTCTTCTC \\
\hline INRA6 & AGGAATATCTGTATCAACCTCAGTC & CTGAGCTGGGGTGGGAGCTATAAATA \\
\hline MM12 & CAAGACAGGTGTTTCAATCT & ATCGACTCTGGGGATGATGT \\
\hline HSC & CTGCCAATGCAGAGACACAAGA & GTCTGTCTCCTGTCTTGTCATC \\
\hline McM527 & GTCCATTGCCTCAAATCAATTC & AAACCACTTGACTACTCCCCAA \\
\hline SRCRSP8 & TGCGGTCTGGTTCTGATTTCAC & CCTGCATGAGAAAGTCGATGCTTAG \\
\hline OarFCB48 & GAGTTAGTACAAGGATGACAAGAGGCAC & GACTCTAGAGGATCGCAAAGAACCAG \\
\hline BM1329 & TTGTTTAGGCAAGTCCAAAGTC & AACACCGCAGCTTCATCC \\
\hline OarFCB 11 & GGCCTGAACTCACAAGTTGATATATCTATCAC & GCAAGCAGGTTCTTTACCACTAGCACC \\
\hline OarFCB304 & CCCTAGGAGCTTTCAATAAAGAATCGG & CGCTGCTGTCAACTGGGTCAGGG \\
\hline INRA23 & GAGTAGAGCTACAAGATAAACTTC & TAACTACAGGGTGTTAGATGAACTC \\
\hline MAF209 & GATCACAAAAAGTTGGATACAACGTGG & TCATGCACTTAAGTATGTAGGATGCTG \\
\hline MAF65 & AAAGGCCAGAGTATGCAATTAGGAG & ССАСТССТССТGAGAATATAACATG \\
\hline
\end{tabular}


Tabela 2 - Múltiplex dos loci, tamanhos dos fragmentos, fluorocromos e temperatura de anelamento utilizados Table 2 - Loci multiplex, size range, fluorescence and annealing temperature

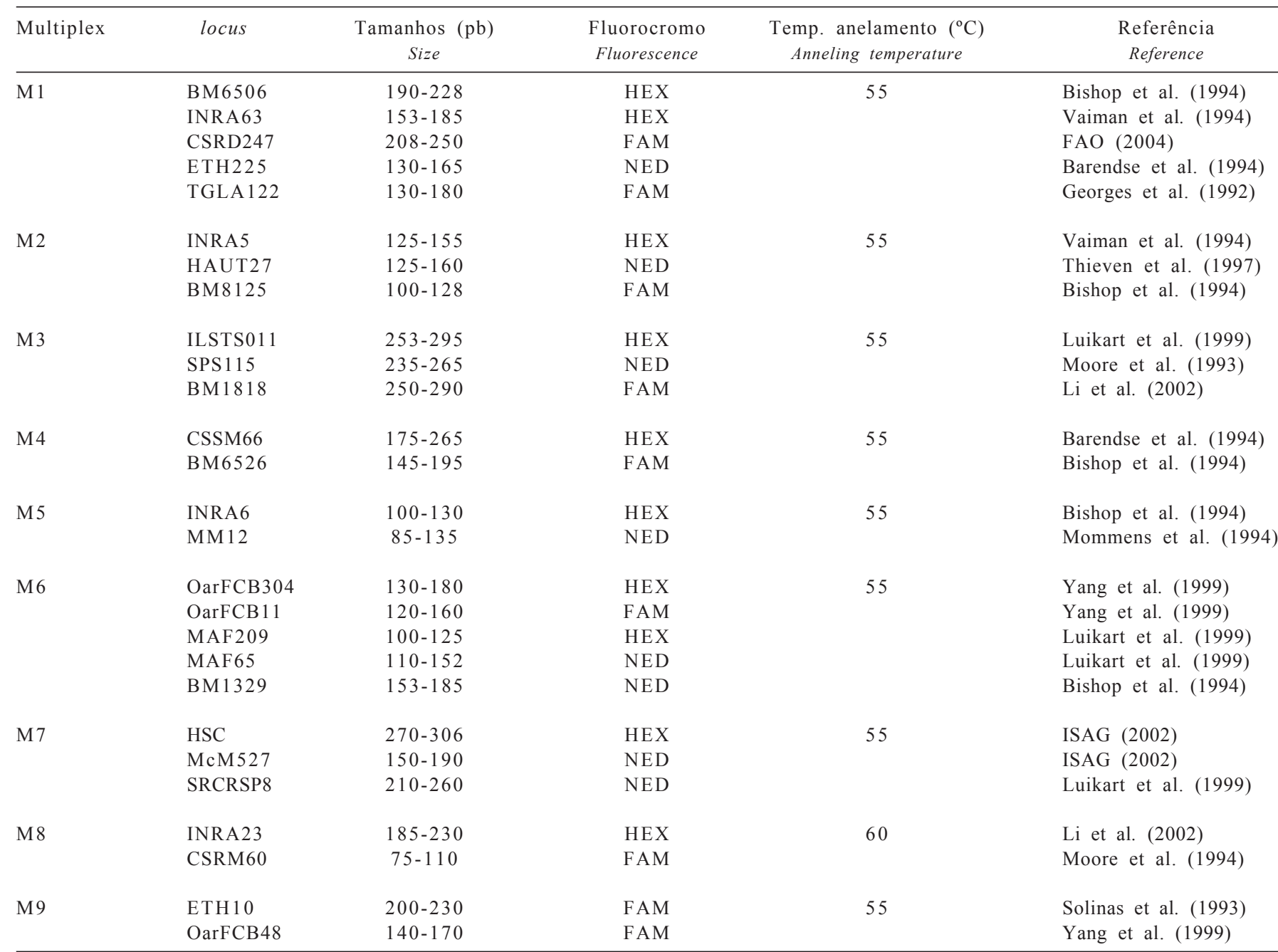

dNTPs e 0,25 mM de primers. Após o preparo, as reações foram levadas a um termociclador e submetidas às seguintes condições de amplificação: 10 minutos a $94^{\circ} \mathrm{C}$, seguidos de 35 ciclos de $94^{\circ} \mathrm{C}$ por 30 segundos, $55^{\circ} \mathrm{C}$ para a maioria dos marcadores e a $60^{\circ} \mathrm{C}$ para os marcadores CSRM60 e INRA 23 por 45 segundos e $72^{\circ} \mathrm{C}$ por 30 segundos. Finalmente, após os 35 ciclos, acrescentaram-se 10 segundos a $72^{\circ} \mathrm{C}$.

Os produtos amplificados foram submetidos à eletroforese em gel de poliacrilamida, em um seqüenciador automático ABI 377XL (Applied Biosystems, Foster City, California, USA). Os resultados das análises de fragmentos e a tipificação alélica foram obtidos por meio dos programas informáticos Genescan Analysis versão 3.7 e Genotyper 2.5 , respectivamente.

O cálculo das freqüências alélicas foi feito com base na contagem direta dos alelos encontrados. Assumindo que existe um estado ideal de equilíbrio Hardy-Weinberg (EHW), a variância de uma freqüência alélica pode ser descrita pela expressão binominal: $\sigma_{x}^{2}=\frac{x(1-x)}{2 n}$ em que $\mathrm{x}$ é a freqüência alélica e n, o número de indivíduos das amostras.

A heterozigosidade pode ser considerada uma medida de variabilidade genética. Considera-se um locus polimórfico quando o alelo mais comum tem freqüência inferior a 0,95 . A heterozigosidade de um marcador é a probabilidade de um indivíduo ser heterozigoto no locus marcador e depende do número de alelos e de sua freqüência na população. A heterozigosidade observada é a proporção de indivíduos heterozigotos nas amostras da população. A heterozigosidade média esperada ou a diversidade genética de um locus é equivalente à heterozigosidade observada, considerando-se apenas as populações em completo equilíbrio. As frequências alélicas e as heterozigosidades foram calculadas por meio do programa Genetix versão 4.01, proposto por Belkhir et al. (1999). 
O Conteúdo de Informação Polimórfica (PIC), descrito por Botstein et al. (1980), é um indicador da qualidade do marcador em estudos genéticos (segregação, identificação de populações e controle de paternidade). Segundo a classificação de Botstein et al. (1980), marcadores com valores de PIC superiores a 0,5 são considerados muito informativos, com valores entre 0,25 e 0,50 mediamente informativos, e com valores inferiores a 0,25 , pouco informativos.

A lei de Hardy-Weinberg foi verificada por Hardy e Weinberg, independentemente, em 1908. Segundo esses autores, em uma população grande, com acasalamento aleatório, sem seleção, mutação ou migração, as freqüências alélicas e genotípicas permanecem constantes de geração a geração, considerando-se uma população em completo equilíbrio.

A constituição genética de uma população é descrita pela especificação e a proporção dos diferentes alelos em cada locus. Os desvios do EHW podem originar-se de fatores como acasalamentos direcionados, subdivisões dentro das populações; coancestrais e antepassados comuns; seleção natural; migração ou fluxo de genes a partir de uma população externa; técnica de amostragem incorreta; e presença de alelos nulos não detectáveis experimentalmente.

Qualquer desvio significativo do EHW indica que a população está subdividida, que existe uma endogamia significativa ou fluxo de genes de outra população. Para o teste de equilibrio de Hardy-Weinberg, utilizou-se o programa Genepop versão 3.4, aplicando-se o método de cadeia de Markov.

\section{Resultados e Discussão}

Os valores de polimorfismo dos loci analisados são descritos na Tabela 3. Os marcadores (TGLA122, MM12, CSSM60, BM6526, HSC e BM8125) apresentaram elevado grau de heterozigosidade média observada, com valores superiores a 70\%. Segundo Ott (1992), um marcador é considerado altamente polimórfico quando apresenta heterozigosidade maior que $70 \%$. No entanto, os marcadores MAF209, BM6506, SPS115, ETH225, INRA63, HAUT27, ETH10 e INRA23 apresentaram nível reduzido de heterozigosidade, com valores inferiores a $50 \%$. Para heterozigosidade média esperada, na maioria dos loci (85\%), observou-se valor superior a $50 \%$, indicando elevada variabilidade genética dos marcadores analisados. A qualidade dos marcadores foi confirmada pelo conteúdo de informação polimórfica, sendo verificados valores superiores a $50 \%$ em mais de $75 \%$ dos loci analisados.
Tabela 3 - Número de alelos (NA), heterozigosidades observada (Ho) e esperada ( $\mathrm{He}$ ) e Conteúdo de Informação Polimórfica (PIC), em função dos loci analisados

Table 3 - $\quad$ Number of alleles (AN), observed heterozygosity (Ho), expected herozygosity (He), Polymorphyc Information Content (PIC) of each analyzed Loci

\begin{tabular}{lcccc}
\hline Locus & NA & Hо & He & PIC \\
\hline CSSM66 & 23 & 0,680 & 0,819 & 0,908 \\
OarFCB304 & 16 & 0,669 & 0,795 & 0,777 \\
MM12 & 14 & 0,733 & 0,752 & 0,724 \\
BM6526 & 14 & 0,716 & 0,744 & 0,724 \\
INRA6 & 13 & 0,638 & 0,847 & 0,830 \\
HSC & 11 & 0,731 & 0,789 & 0,761 \\
BM1329 & 11 & 0,678 & 0,741 & 0,698 \\
OarFCB11 & 11 & 0,659 & 0,687 & 0,643 \\
MAF65 & 11 & 0,508 & 0,717 & 0,675 \\
CSRM60 & 10 & 0,720 & 0,755 & 0,718 \\
BM6506 & 10 & 0,371 & 0,406 & 0,391 \\
BM1818 & 10 & 0,671 & 0,761 & 0,737 \\
TGLA122 & 10 & 0,754 & 0,829 & 0,807 \\
CSRD247 & 09 & 0,666 & 0,748 & 0,717 \\
HAUT27 & 09 & 0,461 & 0,479 & 0,436 \\
SRCRSP8 & 09 & 0,675 & 0,776 & 0,742 \\
INRA23 & 09 & 0,495 & 0,666 & 0,612 \\
INRA5 & 08 & 0,623 & 0,709 & 0,667 \\
OarFCB48 & 08 & 0,598 & 0,632 & 0,572 \\
ILSTS011 & 07 & 0,524 & 0,786 & 0,752 \\
McM527 & 07 & 0,631 & 0,699 & 0,651 \\
BM8125 & 06 & 0,713 & 0,769 & 0,733 \\
INRA63 & 06 & 0,454 & 0,572 & 0,480 \\
SPS115 & 05 & 0,412 & 0,503 & 0,398 \\
ETH10 & 05 & 0,493 & 0,650 & 0,576 \\
ETH225 & 04 & 0,416 & 0,483 & 0,383 \\
MAF209 & 03 & 0,037 & 0,043 & 0,042 \\
\hline
\end{tabular}

Todos os 27 microssatélites analisados foram polimórficos para caracterização genética de raças caprinas brasileiras. Os resultados de número de alelos obtidos por locus analisado estão de acordo com o número mínimo de alelos sugerido por Barker (1994), à exceção do MAF209. Segundo esse autor, número inferior a quatro alelos por locus reduz o efeito sobre o erro-padrão para cálculos de distância genética entre populações. O locus MAF209 é um marcador de ovinos e, apesar de amplificar bem em caprinos, este locus tem se mostrado pouco polimórfico para essa espécie (como encontrado neste estudo), com apenas três alelos, resultado condizente com os encontrados por Luikart et al. (1999), em cabras espanholas. Possivelmente, este locus encontra-se em uma região com baixa variabilidade genética em caprinos.

Por outro lado, o marcador CSSM66 apresentou elevado número médio de alelos (23). Essa quantidade elevada pode ser considerada um número excessivo de alelos, que não é interessante, do ponto de vista prático, em análise de tificação alélica, por dificultar a interpretação dos resultados. Possivelmente, este locus encontra-se em uma região hiperváriavel do DNA no genoma dos caprinos. 
Os valores referentes ao número médio de alelos foram semelhantes aos reportados por alguns autores (Araújo, 2004; Saitbekova et al., 1999; Li et al., 2002; Yang et al., 1999), em estudos sobre a variabilidade genética em caprinos demonstra alto nível de polimorfismo dos loci analisados. Em contraste, Maudet et al. (2002), em estudo com microssatélites em cabras Alpinas Ibex, verificaram número médio de alelos por loci variando de 2 (ILSTS029) a 6 (SRCSRP-24), que indica baixo nível de polimorfismo dos loci em cabras selvagens.

Em análise global, os valores de heterozigosidade média observada por locus revelaram elevado grau de polimorfismo, com valores superiores a 0,5 em mais de $70 \%$ dos loci analisados. Em estudos de diversidade genética em cabras asiáticas, Barker et al. (2001) verificaram alto nível de heterozigosidade em 17 microssatélites analisados, enquanto Saitbekova et al. (1999) observaram heterozigosidade total de 15 (ETH225) a 87\% (ILSTS030) em cabras Suíças, Ibex e Benzoar, com elevada variação entre $l o c i$.

O marcador CSSM66 pode ser considerado altamente informativo ( $\mathrm{PIC}=0,908$ ). No entanto, o PIC depende do número de alelos e de suas freqüências. Desta forma, como comentado anteriormente, o locus com número médio de 23 alelos pode diminuir a eficiência das análises, enquanto, para classificação de Botstein et al. (1980), este marcador seria considerado o mais informativo. Portanto, a informação que este parâmetro produz não deve ser o único aspecto para seleção ou descarte de um marcador ou descartá-lo (Moazami-Goudarzi et al., 1994).

Apenas seis marcadores apresentaram valores de PIC inferior a 50\%, com cinco microssatélites considerados medianamente informativos (ETH225; BM6506; SSP115; HAUT227 e INRA63) e apenas um pouco informativo (MAF209). Estes valores de PIC estão de acordo com os encontrados por Yang et al. (1999), Li etal. (2002) e Jandurová et al. (2004).

O equilíbrio de Hardy-Weinberg (EHW) dos loci para cada raça caprina brasileira encontra-se descrito na Tabela 4. A raça Moxotó foi a que apresentou o maior número de locus em desequilíbrio (11), seguida pela Serrana Azul, Marota e Repartida (9), Canindé (8) e Graúna (7). Em geral, verificou-se diferença de apenas 4 loci em desequilíbrio entre as raças estudadas, demonstrando reduzidos desequilibrio de $\mathrm{HW}$ para os microssatélites analisados. Entretanto, estes números reduzidos de microssatélites em desequilíbrios podem ser esperados

Tabela 4 - Equilibrio de Hardy-Weinberg nas populações estudadas Table 4 - Hardy-Weinberg equilibrium of loci in the studied populations

\begin{tabular}{|c|c|c|c|c|c|c|}
\hline locus & S. Azul & Moxotó & Marota & Canindé & Repartida & Graúna \\
\hline BM6506 & 1,000 & 1,000 & 0,762 & 0,378 & 0,238 & 0,275 \\
\hline BM8125 & 0,209 & 0,145 & 0,230 & 0,866 & 0,618 & 0,603 \\
\hline BM1818 & 0,627 & 0,181 & 0,209 & 0,844 & 0,130 & 0,322 \\
\hline CSRD247 & 0,295 & 0,248 & 0,346 & 0,776 & $0,099 *$ & 0,436 \\
\hline ЕТН 225 & 0,596 & 0,461 & 1,000 & 0,658 & $0,000 *$ & $0,053^{*}$ \\
\hline HAUT 27 & 0,745 & 0,294 & 0,173 & 0,169 & $0,024 *$ & 0,106 \\
\hline ILSTS011 & 0,258 & $0,000 *$ & 0,922 & $0,000 *$ & 0,943 & 0,248 \\
\hline INRA63 & $0,079 *$ & 0,518 & 0,827 & $0,006 *$ & $0,094 *$ & 0,233 \\
\hline INRA5 & $0,014 *$ & 0,420 & $0,087 *$ & 0,905 & 0,606 & 0,914 \\
\hline SPS 115 & $0,072 *$ & $0,000 *$ & 0,750 & $0,002 *$ & $0,005^{*}$ & 0,773 \\
\hline TGLA 122 & 0,130 & 0,311 & 0,200 & 0,220 & 0,273 & 0,130 \\
\hline BM6526 & $0,000 *$ & $0,080 *$ & 0,923 & 0,138 & 0,311 & 0,357 \\
\hline CSRM60 & 0,369 & 0,841 & $0,039 *$ & $0,071 *$ & 0,771 & 0,392 \\
\hline CSSM66 & 0,133 & 0,596 & 0,364 & 0,032 & $0,001 *$ & $0,002 *$ \\
\hline ETH 10 & 1,000 & 0,107 & 0,386 & 0,170 & $0,019 *$ & $0,067 *$ \\
\hline INRA6 & $0,000^{*}$ & $0,062 *$ & 0,231 & $0,065 *$ & 0,664 & $0,000 *$ \\
\hline MM 12 & 0,560 & $0,025^{*}$ & 0,298 & 0,734 & 0,273 & 0,842 \\
\hline HSC & 0,230 & $0,013 *$ & $0,047 *$ & 0,874 & $0,086^{*}$ & 0,185 \\
\hline McM527 & 0,594 & $0,026^{*}$ & 0,686 & 0,606 & 0,047 & 0,662 \\
\hline SRCRSP8 & 0,106 & $0,022 *$ & $0,093 *$ & $0,075 *$ & 0,869 & $0,012 *$ \\
\hline OarFCB48 & $0,056^{*}$ & 0,403 & $0,043 *$ & 0,307 & 0,355 & 0,468 \\
\hline BM1329 & 0,951 & 0,347 & 0,849 & 0,419 & 0,574 & 0,160 \\
\hline OarFCB 11 & 0,206 & $0,032 *$ & $0,085 *$ & 0,174 & 0,718 & 0,127 \\
\hline OarFCB304 & 0,636 & 0,500 & 0,001 & 0,161 & 0,298 & 0,337 \\
\hline INRA23 & $0,016^{*}$ & 0,137 & $0,000 *$ & $0,000 *$ & 0,240 & $0,000 *$ \\
\hline MAF209 & $0,000 *$ & $0,000^{*}$ & $0,000 *$ & 1,000 & $0,051^{*}$ & $0,000 *$ \\
\hline MAF65 & $0,000^{*}$ & $0,000^{*}$ & $0,003 *$ & 0,204 & 0,148 & $0,017 *$ \\
\hline Total & 9 & 11 & 9 & 7 & 9 & 7 \\
\hline
\end{tabular}

* loci em desequilibrio $(P>0,10)$.

* loci in desequilibrium $(P>0.10)$. 
tanto em populações de raças comercias com acasalamentos dirigidos, permitindo aumento na frenquência de determinados individuos, como em raças ameaçadas de extinção (objeto deste estudo) e podem ser explicados por diverdos fatores: subdivisões dentro das populações, antepassados comuns, seleção natural, migração ou fluxo de genes a partir de população externa.

Verificou-se que os desequilíbrios de Hardy-Weinberg para os marcadores alternaram-se dentro das populações e que a maioria dos marcadores (aproximadamente 70\%) está equilibrada, demonstrando a importância dos marcadores microsstélites para identificação e avaliação da variabilidade genética em estudos com raças caprinas brasileiras.

\section{Conclusões}

Os microssatélites analisados são polimórficos e com alta capacidade para identificar a variabilidade e a diversidade genética em caprinos, à exceção dos marcadores MAF209 e CSSM66. Estes microssatélites podem ser utilizados para caracterização genética de raças caprinas brasileiras, auxiliando em programas de conservação e melhoramento dos recursos genéticos do país.

\section{Literatura Citada}

ARAÚJO, A.M. Paternidade e diversidade genética em caprinos no Brasil por meio de microssatélites de DNA. Viçosa, MG: Universidade Federal de Viçosa, 2004. 90p. Tese (Doutorado em Genética e Melhoramento). Universidade Federal de Viçosa, 2004. 90p.

BARKER, J.S.F.; TAN, S.G.; MOORE, S.S. et al. Genetic variation within and relationship among populations of Asian goats (Capra hircus). Journal of Animal Breeding Genetics, v.118, p.213-233, 2001.

BARENDSE, W.; ARMITAGE, S.M. A genetic linkage map of the bovine genome. Nature Genetics, v.6, p.227-235, 1994.

BELKHIR, K. Genetix: Logiciel sous Windows ${ }^{\mathrm{TM}}$ pour la génétique des populations. Laboratoire Génome, Populations, Interactions. CNRS UPR 9060, 1999.

BISHOP, M.D.; KAPPES, S.M. A genetic linkage map for cattle. Genetics, v.136, p.619-639, 1994

BOTSTEIN, D.; WHITE, R.L.; SKOLMICK, H. et al. Construction of a genetic linkage map in man using restriction fragment lenght polymorphisn. American Journal of Human Genetics, v.32, p.314-331, 1980.

FAO. Secondary guidelines for developmen of National Farm Animal Genetic Resources Management Plans: Measurement of Domestic Animal Diversity (MoDAD): Recommended Microsatellite Markers. Rome: FAO. 2004.
GAMA, L.T. Manutenção da variabilidade genética em programas de seleção. In: SIMPÓSIO INTERNACIONAL DE CONSERVAÇÃO DE RECURSOS GENÉTICOS (RAÇAS NATIVAS PARA O SEMI-ÁRIDO), 1., 2004, Recife. Anais... Recife: 2004. p.38-44.

GEORGES, M.; MASSEY, J.M. Polimorphic DNA markers in Bovidae. Patent WO 92/13102, 1992.

ISAG. Disponível em: http://www.isag.org.uk Acesso em: 15/12/2002. JANDUROVÁ, O.M.; KOOT, T.; IKOTTOVÁ, B. et al. Seven microsatellites markers useful for determining genetic variability in White and Brown Short-Haired goat breeds. Small Ruminant Research, 2004 (in press).

LI, M.H.; ZHAO, S.H.; BIAN, C. et al. Genetic relationships among twelve Chinese indigenous goat populations based on microsatellite. Genetics Selection and Evolution, v. 34 p.729-744, 2002.

LUIKART, G.; BIJU-DUVAL, M-P.; ERTUGRUL, O. et al. Power of 22 microssatelite markers in fluorescent multiplexes for parentage testing in goats (Capra hircus). Animal Genetics v.30, p.431-438, 1999

MAUDET, C.; MILLER, C.; BASSANO, B. et. al. Microsatellite DNA and recent statistical methods in wildlife conservation management: applications in Alpine ibex (capra ibex ibex). Molecular Ecology, v.11, p.421-436, 2002.

MOAZAMI-GOUDARZI, K.; VAIMAN, D.; MERCIER, D. et al. Emploi de microsatellites pour l'analyse de la diversité genétique des races bovines francaises: premiers resultants. Genetics Selection and Evolution, v.26, p.155-165, 1994.

MOMMENS, G.W.; COPPIETERS, A. Dinucleotide repeat polymorphism at the bovine MM12E6 and MM8D3 loci. Animal Genetics, v.25, p.368, 1994

MOORE, S.S.; BYRNE, K. Dinucletotide polymorphism at the bovine calmodulin independent adenylcyclase locus. Animal Genetics, v.24, p.150, 1993.

MOORE, S.S.; BYRNE, K. Characterization of 65 bovine microsatellites. Mammalian Genome, v.5, p.84-90, 1994.

OTT, J. Strategies for characterizing highly polymorphic markers in human gene mapping. American Journal of Human Genetics, v.51, p.283-290, 1992.

SAITBEKOVA, N.; GAILLARD, C.; RUFF, G.O. et al. Genetic diversity in Swiss goat breeds based on microsatellite analysis. Animal Genetics, v.30, p.36-41, 1999.

SOLINAS-TOLDO, S.; FRIES, R. Physically mapped, cosmidderived microsatellite markers as anchor loci on bovine chromosomes. Mammalian Genome, v.4, p.720-727, 1993.

THIEVEN, U.; SOLINAS-TOLDO, S. Polymorphic CAmicrosatellites for the integration of the bovine genetic and physical map. Mammalian Genome, v.8, p.52-55, 1997.

VAIMAN, D.; MERCIER, D. A set of 99 cattle microsatellites: characterisation, synteny mapping, and polymorphism. Mammalian Genome, v.5, p.288-297, 1994.

YANG, L.; ZHAO S.H.; LI, K. et al. Determination of relationships among five indigenous Chinese goat breeds with six microsatellite markers. Animal Genetics, v.30, p.452-456, 1999.

WALSH, P.S.; METZGER, D.A.; HIGUCHI, R. Chelex 100 as a medium for a simple extraction of DNA for PCR-based typins from forensic material. Biotecchniques, v.10, p.506-513, 1991. 